\title{
Geographical Prevalence of Isolated Patellofemoral osteoarthritis
}

\author{
Heta Patel* \\ Assistant Professor, Ashok and Rita Patel Institute of Physiotherapy, Charusat, India
}

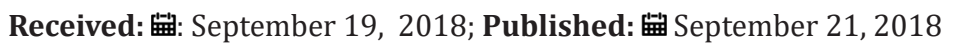

*Corresponding author: Heta Patel, Assistant Professor, Ashok and Rita Patel Institute of Physiotherapy, Charusat, India

\begin{abstract}
Mini Review
Osteoarthritis is not a single disease [1]. Osteoarthritis affects isolated Tibiofemoral compartment, isolated patellofemoral compartment or the both combined of them. Among various approaches to sub classification of knee osteoarthritis, European League against Rheumatism (EULAR) Task Force recognized subtype of compartmental distribution for diagnosis of osteoarthritis [2]. Traditionally, in cases of osteoarthritis of knee most of the research has focused on understanding of general osteoarthritis of knee or tibiofemoral osteoarthritis of knee. In fact, isolated patellofemoral osteoarthritis may not be so rare. Even though, the PF compartment is rarely considered in investigation of osteoarthritis.
\end{abstract}

\section{Need of the Review}

Identifying the prevalence of the condition is a milestone for the identification of risk factors, these are directly related to the culture, ethnicity and the lifestyle they have and that makes it easy to decide on the treatment aspect. Current treatment paradigms for Patellofemoral osteoarthritis are inadequate. Furthermore, published guidelines are based exclusively on research utilizing Tibiofemoral osteoarthritis; there is no evidence for Patellofemoral arthritis. In many regions where data is lacking, in future we could have studies mentioning the prevalence rates and also determine the relevance of ethnicity.

\section{Review Question}

What is the Geographic Prevalence of Patellofemoral osteoarthritis?

\section{Objective}

To determine the geographical proportion of Patellofemoral osteoarthritis in the world.

\section{Methodology}

\section{Key Words Used for Search}

The Terms Associated with Search: Osteoarthritis, Isolated Patellofemoral joint, Knee, Prevalence rate of Isolated Patellofemoral arthritis, Biomechanics of Patellofemoral joint.

\section{Search Strategy: Terms using AND, OR \& NOT}

Data Based Searched: Computerized searches from Pubmed, EBSCO, Google scholar, Science Direct, Research Gate, Scopus. There were no language, Gender and Age limit.

\section{Definition}

Patellofemoral arthritis occurs due to the loss of cartilage of patella and the trochlear groove of the femur. Mc Alindon TE [3] studied on Radiographic patterns of osteoarthritis of the knee joint in the community and suggested if cases where both medial and lateral facets are involved, the lateral facet usually has worse disease [3].

\section{Biomechanics of Patellofemoral Joint}

Patellofemoral joint is the most incongruent joint in the body. Although the patella enhances the tibiofemoral mechanisms, the characteristics, responses and problems of the patellofemoral joint are distinct enough from the tibiofemoral joint to warrant separate attention.

\section{Geographical Area Distribution}

There is geographical variability may be explained by the different age ranges evaluated by different studies. These findings were consistent with Pereira et al. [4], who found that studies conducted in Europe, particularly Northern Europe, reported lower prevalence of knee osteoarthritis [4]. Lower prevalence of patellofemoral osteoarthritis in the Northern European studies. Body mass index of included participants could also explain geographical variation of patellofemoral osteoarthritis prevalence.

A high prevalence of patellofemoral osteoarthritis was observed in studies conducted in the Middle East and Asia, whereas lower prevalence rates were reported in Estonia and Sweden. The two studies from Estonia (35-57 years) and Sweden (35-54 years) evaluated younger cohorts with smaller age ranges than the studies from Saudi Arabia (20-93 years) and Turkey (44-76 years) [5-7]. We could also speculate that ethnicity could be a contributing factor to variability in the point prevalence of patellofemoral osteoarthritis 
across geographical regions. A previous study has suggested that the culture of kneeling, particularly in Asian countries could lead to high prevalence of knee osteoarthritis [8]. However; there has been no conclusive evidence to suggest any cultural link with patellofemoral OA. In fact, conflicting evidence has suggested that the increased contact area between the patella and femoral trochlear during kneeling could be a protective mechanism from developing patellofemoral osteoarthritis [9].

Whereas a previous epidemiological study reported that the prevalence of radiographic osteoarthritis increased with age, particularly in people aged 55 years or more. A literature review has shown that certain genes have high associations with knee osteoarthritis in some ethnic populations but do not have the same effect on others [10]. For example, a variant in expression of a gene responsible for cartilage and bone growth was shown to increase risk of developing knee osteoarthritis in Asian cohorts (Japanese, Chinese and Korean), but not in European cohorts [10]. We could therefore speculate that ethnic groups have different genetic predispositions for developing osteoarthritis, which may explain the variability of patellofemoral osteoarthritis prevalence across different geographical regions.

However, current treatment paradigms for Patellofemoral osteoarthritis are inadequate. Furthermore, published guidelines are based exclusively on research utilizing Tibiofemoral osteoarthritis, no evidence for Patellofemoral arthritis. It is inappropriate to assume that treatments designed for Tibiofemoral osteoarthritis are optimal for Patellofemoral osteoarthritis.

\section{Conclusion}

Ethnicity does play a role in the prevalence of patellofemoral arthritis. Once geographical prevalence is identified only then it is possible to further progress towards its prevention aspect and rehabilitation aspect of patellofemoral arthritis. Future epidemiological studies could help us provide the data lacking in various regions.

\section{References}

1. Lane NE, Brandt K, Hawker G, Peeva E, Schreyer E, et al. (2011) OARSIFDA initiative: defining state of osteoarthritis. Osteoarthritis Cartilage 19(5): 478-482.

2. Zhang W, Doherty M, Peat G, Bierma-Zeinstra MA, Arden NK, et al. (2010) EULAR evidence based recommendations for the diagnosis of knee osteoarthritis. Ann Rheum Dis 69(3): 483-489.

3. McAlindon TE, S Snow, C Cooper, Dieppe PA (1992) Radiographic patterns of osteoarthritis of the knee joint in the community: The importance of the patellofemoral joint. Ann Rheum Dis 51(7): 844-849.

4. Pereira D, Peleteiro B, Araújo J, Branco J, Santos RA, et al. (2011) The effect of osteoarthritis definition on prevalence and incidence estimates: A systematic review. Osteoarthritis Cartilage 19: 1270-1285.

5. Felson DT, Naimark A, Anderson J, Kazis L, Castelli W, et al. (1987) The prevalence of knee osteoarthritis in the elderly. The Framingham Osteoarthritis Study. Arthritis Rheum 30(8): 914-918.

6. Cicuttini FM, Spector T, Baker J (1997) Risk factors for osteoarthritis in the tibiofemoral and patellofemoral joints of the knee. J Rheumatol 24(6): 1164-1167.

7. Spector TD, Harris PA, Hart DJ, Cicuttini FM, Nandra D, et al. (1996) Risk of osteoarthritis associated with long-term weight-bearing sports: A radiologic survey of the hips and knees in female ex-athletes and population controls. Arthritis Rheum 39(6): 988-995.

8. Muraki S, Oka H, Akune T, Mabuchi A, En-yo Y, et al. (2009) Prevalence of radiographic knee osteoarthritis and its association with knee pain in the elderly of Japanese population-based cohorts: the ROAD study. Osteoarthritis Cartilage 17(9): 1137-1143.

9. Rytter S, Egund N, Jensen LK, Bonde JP (2009) Occupational kneeling and radiographic tibiofemoral and patellofemoral osteoarthritis. J Occup Med Toxicol.

10. Valdes AM, Spector TD (2011) Genetic epidemiology of hip and knee osteoarthritis. Nat Rev Rheumatol 7(1): 23-32.

\section{ISSN: 2574-1241}

DOI: 10.26717/BJSTR.2018.09.001772

Heta Patel. Biomed J Sci \& Tech Res

CC (i) This work is licensed under Creative Commons Attribution 4.0 License

Submission Link: https://biomedres.us/submit-manuscript.php

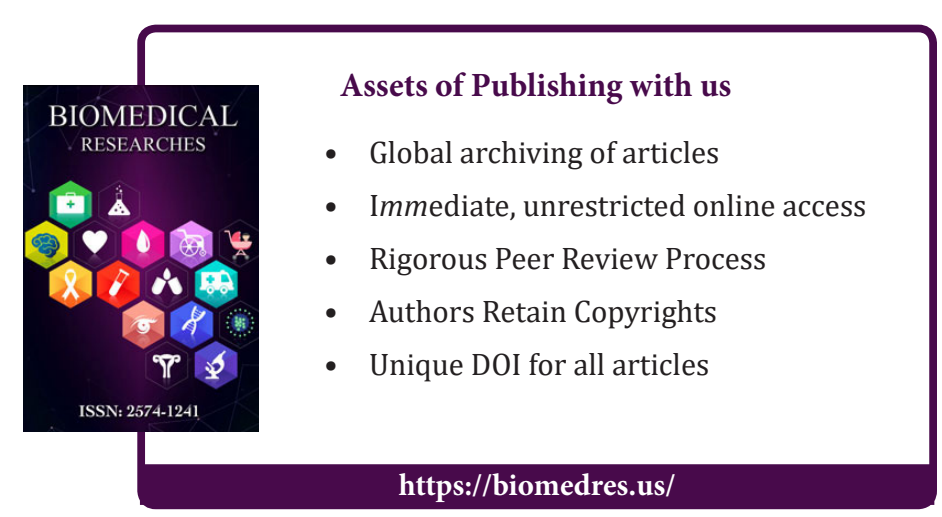

